\title{
Vivência das Mulheres Atendidas por Alunos de Medicina em Consulta Ginecológica
}

\author{
Experience of Women Attended by Medical \\ Students in Gynecological Care
}

\author{
Suzana Maria Pires do Rio ${ }^{l}$ \\ Ivana Mares Trivellato \\ Natália Mares Caldeiral \\ Silvia Ferreira Araujo ${ }^{I}$ \\ Dilermando Fazito de Rezende ${ }^{l}$
}

\section{PALAVRAS-CHAVE:}

- Estudantes;

- Ginecologia;

- Atendimento Médico;

- Assistência ao Paciente;

- Educação Médica;

\section{KEYWORDS:}

- Students;

- Gynecology;

- Medical Care;

- Patient Care;

- Medical Education;

Recebido em: 10/12/2012

Reencaminhado em: 06/06/2013

Aprovado em: 07/10/2013

\section{RESUMO}

O aprendizado por meio da interação com pacientes é vital para a educação médica. É sabido que a participação do aluno na prática médica é bem aceita pelos pacientes. Entretanto, na especialidade de Ginecologia, a natureza íntima da consulta torna a discussão do tema mais delicada. Este trabalho avaliou como as mulheres atendidas em ambulatórios do SUS percebem o atendimento ginecológico prestado por estudantes de Medicina e objetivou identificar pontos positivos e negativos da consulta relatados por elas. Foi feito um estudo de corte transversal com 360 mulheres através da realização de entrevistas. Os resultados apontaram que, embora a maioria das mulheres considerasse boa a presença do estudante, 52,7\% relataram receio quanto à presença deste. A principal justificativa para o sentimento positivo foi o interesse do aluno. Dentre as que julgaram ruim a presença do estudante, a vergonha foi o principal motivo citado. A maioria avaliou que o sexo do aluno não influencia sua opinião sobre a consulta. Concluiu-se que a avaliação de como as mulheres vivenciam esse atendimento é essencial para aprimorar a relação estudante-paciente e aumentar a aceitação desse tipo de atendimento.

\section{ABSTRACT}

Learning through interaction with patients is vital for medical education. It is well established that the participation of the student in medical practice is well accepted by patients. However, in the specialty of gynecology, the intimate nature of the consultation makes the discussion of the topic more delicate. This study evaluated how women attending public clinics view gynecological care provided by medical students and intended to identify the strengths and weaknesses of the consultations they reported. This cross-sectional study was conducted through questionnaires answered by 360 women. The results showed that although most women consider student presence as a good thing, 52.7\% reported fear of their presence. The main reason for the positive feelings was the student's interest. Among those who viewed it as a negative thing, feeling embarrassed was the main reason cited. The majority found that student gender does not influence in their opinion about the consultation. In conclusion, the assessment of how women experience this care is essential to enhance the student-patient relationship and increase acceptance of this type of care. 


\section{INTRODUÇÃO}

Aprender, por meio da interação e contato direto com pacientes, é vital para a educação médica ${ }^{1-4}$. As Diretrizes Curriculares Nacionais do Curso de Graduação em Medicina recomendam que a estrutura do curso insira o aluno precocemente em atividades práticas ${ }^{5}$. O objetivo é promover interação ativa do acadêmico com usuários e profissionais de saúde desde o início de sua formação, para que este lide com problemas reais e assuma responsabilidades crescentes como agente prestador de cuidados e atenção ${ }^{5}$. Nesse contexto, o estudante vem tomando lugar na comunidade.

A presença do aluno na prática médica, em geral, é bem aceita pelos pacientes nas diversas áreas da Medicina ${ }^{1,6}$. Entretanto, na especialidade de Ginecologia e Obstetrícia, a natureza íntima da consulta e do exame torna a discussão do tema mais delicada ${ }^{2}$. O conhecimento dos fatores que influenciam a permissão das pacientes quanto à participação dos estudantes no seu cuidado tem recebido pouca atenção $0^{2,7}$. A elucidação de aspectos que incentivam a participação dos pacientes na educação dos futuros médicos é crucial ${ }^{8}$.

Geralmente, as pacientes são receptivas e ficam satisfeitas com a participação do estudante de Medicina no seu cuidado ${ }^{1,2,3,7,9,10,11}$. A principal motivação para concordar com o envolvimento do estudante é o desejo de contribuir com a formação médica ${ }^{3,7,9}$. O papel das mulheres no ensino e treinamento clínico tem sido ativamente desenvolvido, fazendo delas potenciais parceiras e educadoras efetivas ${ }^{9}$.

Entretanto, existem fatores que podem gerar na mulher relutância em aceitar o envolvimento acadêmico, e essa recusa dificulta o desenvolvimento de habilidades clínicas pelos estudantes ${ }^{7}$. Um dos fatores mais avaliados é o impacto que o sexo do acadêmico pode causar ${ }^{2,4}$.

Alternativas para contornar a barreira existente entre pacientes e acadêmicos foram a implementação do uso de manequins e simulações no ensino médico ${ }^{3,12}$. Apesar disso, não existem substitutos adequados para o contato com pacientes reais ${ }^{8}$, devendo este ser apenas um método complementar. Proporcionar nível adequado de conforto à paciente, em relação à participação do estudante durante o seu cuidado, é elemento-chave para a educação médica ${ }^{3}$. Tornar isso uma prática diária encorajaria as mulheres a aceitarem bem os estudantes e evitaria o uso exclusivo de métodos de ensino alternativos.

É importante compreender os valores que influenciam as mulheres a aceitarem ou não o envolvimento do acadêmico em seu cuidado, com o intuito de aprimorar a inserção do estudante na prática clínica. Estudos continuados desses as- pectos justificam-se pela escassez de trabalhos brasileiros e latino-americanos que abordam o assunto sob a perspectiva da consulta ginecológica.

O presente trabalho objetivou identificar como as pacientes atendidas em ambulatórios do SUS de uma cidade de médio porte de Minas Gerais percebem o atendimento ginecológico prestado por estudantes de Medicina. Isto envolveu avaliar os pontos positivos e negativos da consulta relatados pelas pacientes, a influência do sexo do aluno, o receio ou não desse atendimento e as justificativas apresentadas para esse sentimento.

\section{CASUÍSTICA E MÉTODOS}

A presente investigação é um estudo de corte transversal sobre a vivência de mulheres atendidas por estudantes de Medicina em ambulatórios de Ginecologia e Obstetrícia de uma cidade de médio porte do interior de Minas Gerais a respeito da qualidade e características desse atendimento.

O cálculo do contingente amostral baseou-se na variável receio, assumindo-se que a resposta sim à questão "Você receia fazer exame ginecológico na presença de estudantes de Medicina?" seria de 50\%. A precisão da estimativa foi de aproximadamente $6 \%$, e o erro amostral de $5 \%$.

A amostra final foi composta por 360 mulheres, escolhidas casualmente dentre as que buscaram as unidades básicas de saúde para atendimento ginecológico de novembro de 2010 a junho de 2011. Esse montante foi formado por todas aquelas que estavam presentes na recepção dos consultórios nos momentos em que os autores visitaram as unidades e concordaram em submeter-se ao questionário. Foram excluídas as mulheres com idade inferior a 18 anos, acima de 65 anos e gestantes. Para a coleta dos dados, foram visitados, mediante a conveniência dos pesquisadores, 15 dos 16 postos em que a Faculdade de Medicina local se encontra inserida. No total, o município conta com 23 unidades de saúde.

As mulheres incluídas na pesquisa responderam a um questionário, por intermédio dos autores, que continha questões relativas à percepção das particularidades do atendimento médico realizado pelos estudantes com a supervisão e orientação de docentes (ginecologistas) da Faculdade de Medicina. Em seguida, realizou-se o processamento estatístico das respostas dadas às questões, com o objetivo de determinar relações das características do atendimento com as opiniões das participantes.

O questionário aplicado foi elaborado exclusivamente para a ocasião, e as perguntas foram baseadas em dados bibliográficos validados. Foi composto por questões abertas 
e fechadas relativas a: (1) características sociais e demográficas: idade; escolaridade; número de filhos; (2) sentimentos da mulher em relação à consulta com o estudante; receio; conhecimento da presença do estudante durante a consulta; o sexo do aluno que havia prestado atendimento e se havia preferência por algum; sentimento em relação à presença do estudante (bom, ruim) e o motivo. Para as perguntas: "se você achou bom, por quê?" e "se você achou ruim, por quê?", foi dada à participante a oportunidade de escolher a opção "outras". Quando esta opção era assinalada, a entrevistada tinha a possibilidade de justificar sua escolha. Todas as participantes responderam integralmente às perguntas do questionário.

Para a análise estatística foram construídas as distribuições de frequência e calculadas as médias e desvios padrões (DP) indicados para cada variável. Nas comparações das porcentagens utilizou-se preferencialmente o teste qui-quadrado. Os testes exatos de Fisher, de Student ou Fisher para tabela de Anova foram empregados quando a frequência esperada da variável era menor que 5 . Na sessão de resultados, os valores exibidos na coluna $\mathrm{X}^{2} / \mathrm{F}$ corresponderam aos resultados dos

TABela 1

Comparação das respostas à questão "Você receia fazer o exame ginecológico na presença de estudantes de Medicina?"

\begin{tabular}{|c|c|c|c|c|c|c|}
\hline \multirow{2}{*}{$\begin{array}{l}\text { Questões do } \\
\text { questionário }\end{array}$} & \multicolumn{2}{|c|}{ Resposta Não } & \multicolumn{2}{|c|}{ Resposta Sim } & \multirow[t]{2}{*}{$\mathrm{X}^{2} / \mathrm{F}^{(*)}$} & \multirow[t]{2}{*}{$\mathrm{P}^{(* *)}$} \\
\hline & $\mathrm{N}$ & $\%$ & $\mathrm{~N}$ & $\%$ & & \\
\hline \multicolumn{7}{|c|}{ Você recebeu informação quanto à presença do estudante na consulta? } \\
\hline Não & 92 & 54,1 & 109 & 57,3 & 0,3 & 0,5 \\
\hline Sim & 78 & 45,8 & 81 & 42,6 & & \\
\hline \multicolumn{7}{|c|}{ Se não recebeu informação, você gostaria de ser informada? } \\
\hline Não & 25 & 27,1 & 6 & 5,5 & 17,9 & 0,0 \\
\hline Sim & 67 & 72,8 & 103 & 94,5 & & \\
\hline \multicolumn{7}{|c|}{ Achei a presença do aluno boa. } \\
\hline Não & 41 & 28,4 & 59 & 42,7 & 6,2 & 0,0 \\
\hline $\operatorname{Sim}$ & 103 & 71,5 & 79 & 57,2 & & \\
\hline \multicolumn{7}{|c|}{ Você já fez alguma consulta ginecológica realizada por um estudante de Medicina? } \\
\hline Não & 26 & 15,2 & 52 & 27,3 & 7,7 & 0,0 \\
\hline Sim & 144 & 84,7 & 138 & 72,6 & & \\
\hline \multicolumn{7}{|c|}{ Qual o seu sentimento em relação a esta primeira consulta com a presença do estudante? } \\
\hline Negativo & 15 & 57,7 & 43 & 82,7 & - & 0,0 \\
\hline Neutro & 4 & 15,4 & 2 & 3,9 & & \\
\hline Positivo & 7 & 26,9 & 7 & 13,7 & & \\
\hline \multicolumn{7}{|c|}{ Você tem filhos? } \\
\hline Sim & 152 & 89,4 & 147 & 77,3 & 9,2 & 0,0 \\
\hline Não & 18 & 10,5 & 43 & 22,6 & & \\
\hline \multicolumn{7}{|c|}{ Você preferia ser submetida ao exame sem a presença do aluno? } \\
\hline Não & 79 & 54,8 & 16 & 11,5 & 59,0 & 0,0 \\
\hline Sim & 65 & 45,1 & 122 & 88,4 & & \\
\hline \multicolumn{7}{|c|}{ Você voltaria a consultar sabendo da presença do aluno? } \\
\hline Não & 3 & 2,0 & 42 & 30,4 & 42,2 & 0,0 \\
\hline $\operatorname{Sim}$ & 141 & 97,9 & 96 & 69,5 & & \\
\hline \multicolumn{7}{|c|}{ O sexo do aluno influencia em sua opinião sobre a consulta? } \\
\hline Não & 103 & 71,5 & 50 & 36,2 & 35,3 & 0,0 \\
\hline Sim & 41 & 28,4 & 88 & 63,7 & & \\
\hline
\end{tabular}

(*) Resultado do teste de qui-quadrado de Pearson ou do teste exato de Fisher.

(**) Valor de P. 
testes qui-quadrado de Pearson, sem o ajuste de Yates, ou o teste exato de Fisher para tabelas de contingência. $O$ nível de significância adotado foi de $5 \%$.

No tocante aos aspectos éticos, não houve conflito de interesses, e a pesquisa foi aprovada pelo Comitê de Ética em Pesquisa (CEP) da Unipac (protocolo $n^{\circ} 741 / 06 / 2010$ ). Todas as mulheres que participaram da entrevista assinaram o Termo de Consentimento Livre e Esclarecido.

\section{RESULTADOS}

As características sociais e demográficas da população em estudo foram delineadas e encontrou-se: média de idade de 39,3 anos com desvio-padrão (DP) de 12,0; predomínio de mulheres de baixa escolaridade (fundamental completo: 53,3\%; médio completo: $40,2 \%$, superior: 6,3\%). A maioria possuía filhos, uma média de 2,3 filhos por mulher, com DP =1,5.

Os motivos relatados para a procura do atendimento ginecológico foram agrupados em cinco categorias. O motivo ro- tina foi citado por 207 (57,5\%) mulheres, o motivo distúrbios menstruais correspondeu a $25(6,9 \%)$ mulheres, alterações da mama e climatério a 21 (5,8\%) e 19 (5,3\%), respectivamente, e 88 $(24,4 \%)$ mulheres procuraram atendimento por outros motivos.

No intuito de compreender as características das participantes e as respostas que deram à entrevista, foram constituídos grupos para comparar as frequências das respostas prevalentes em cada situação.

A primeira comparação consistiu em avaliar se a paciente tinha receio de ser atendida por um estudante de Medicina durante o atendimento ginecológico. Apresentaram receio 190 (52,8\%) e observou-se que determinadas variáveis podem estar associadas a esse sentimento. O resultado da análise do impacto dessas variáveis sobre o receio da paciente encontra-se na Tabela 1 .

A segunda análise avaliou o impacto que o sexo do aluno exerceria na aceitação dos estudantes pela paciente. A maioria, $153(54,3 \%)$, afirmou que o sexo do aluno não influenciaria em

TABela 2

Comparação das respostas à questão "Qual o sexo do(s) aluno(s) que realizou seu exame?"

\begin{tabular}{|c|c|c|c|c|c|c|c|c|c|c|}
\hline \multirow{2}{*}{$\begin{array}{l}\text { Questões do } \\
\text { questionário }\end{array}$} & \multicolumn{2}{|c|}{ Masculino } & \multicolumn{2}{|c|}{ Feminino } & \multicolumn{2}{|c|}{ Ambos } & \multicolumn{2}{|c|}{ Não sei } & \multirow[t]{2}{*}{$\mathrm{X}^{2} / \mathrm{F}^{(*)}$} & \multirow[t]{2}{*}{$\mathrm{P}^{(* *)}$} \\
\hline & $\mathrm{N}$ & $\%$ & $\mathrm{~N}$ & $\%$ & $\mathrm{~N}$ & $\%$ & $\mathrm{~N}$ & $\%$ & & \\
\hline
\end{tabular}

Achei bom porque os alunos costumam ser bastante interessados.

$\begin{array}{llllllllll}\text { Não } & 11 & 42,3 & 19 & 57,5 & 52 & 42,6 & 1 & 100 & - \\ \text { Sim } & 15 & 57,6 & 14 & 42,4 & 70 & 57,3 & 0 & 0,0 & 0,2\end{array}$

Achei bom porque recebi mais orientações.

$\begin{array}{lllllllllll}\text { Não } & 13 & 50,0 & 15 & 45,4 & 58 & 47,5 & 1 & 100 & - \\ \text { Sim } & 13 & 50,0 & 18 & 54,5 & 64 & 52,4 & 0 & 0,0 & 0,8\end{array}$

Achei bom porque senti mais conforto quando o aluno era do sexo feminino.

$\begin{array}{lcccccccccc}\text { Não } & 26 & 100 & 13 & 39,3 & 113 & 92,6 & 1 & 100 & - \\ \text { Sim } & 0 & 0,0 & 20 & 60,6 & 9 & 7,3 & 0 & 0,0 & 0,0\end{array}$

Achei bom porque o exame foi mais completo.

$\begin{array}{lllllllllll}\text { Não } & 15 & 57,6 & 27 & 81,8 & 74 & 60,6 & 0 & 0,0 & - \\ \text { Sim } & 11 & 42,3 & 6 & 18,1 & 48 & 39,3 & 1 & 100 & 0,0\end{array}$

Achei bom porque senti mais segurança com mais pessoas realizando meu exame.

\begin{tabular}{|c|c|c|c|c|c|c|c|c|c|c|}
\hline Não & 22 & 84,6 & 30 & 90,9 & 117 & 95,9 & 0 & 0,0 & \multirow[t]{2}{*}{-} & \multirow[t]{2}{*}{0,0} \\
\hline Sim & 4 & 15,3 & 3 & 9,0 & 5 & 4,1 & 1 & 100 & & \\
\hline \multicolumn{11}{|c|}{ Sinto que sou mais bem atendida quando o estudante está participando. } \\
\hline Sim & 14 & 33,3 & 9 & 20,4 & 40 & 20,9 & 0 & 0,0 & \multirow{2}{*}{-} & 0,2 \\
\hline \multicolumn{10}{|c|}{ Achei ruim porque sinto vergonha. } & \\
\hline Sim & 5 & 50,0 & 5 & 83,3 & 42 & 75,0 & 3 & 100 & - & 0,2 \\
\hline
\end{tabular}

(*) Resultado do teste de qui-quadrado de Pearson ou do teste exato de Fisher.

(**) Valor de P. 
sua opinião sobre a consulta. A comparação dos sentimentos das mulheres em relação ao sexo do aluno que prestou atendimento encontra-se na Tabela 2.

$\mathrm{O}$ último aspecto estudou a percepção geral em relação à presença do aluno durante o atendimento. Dentre aquelas que tinham receio de ser atendidas pelo estudante, quando interrogadas se consideravam a presença boa ou ruim, a maior parte, $182(64,5 \%)$, considerou como boa a participação do aluno, e 75 $(26,6 \%)$, como ruim. Ressalta-se que nesta análise as pacientes poderiam responder sim às duas opções, justificando posteriormente cada uma delas. As justificativas encontram-se na Tabela 3.

\section{TABELA 3}

Justificativas às respostas dadas pelas pacientes à pergunta: "O que você achou da presença do aluno durante o exame?"

$$
\text { Questões do questionário }
$$$$
\mathrm{N}
$$$$
\%
$$

Achei bom porque os alunos costumam ser bastante interessados.

$$
\text { Sim } 99
$$

Achei bom porque recebi mais orientações.

Sim 95

Achei bom porque senti mais conforto quando o aluno era do sexo feminino.
Sim
29
16,0

Achei bom porque o exame foi mais completo.

Sim 66

Achei bom porque senti mais segurança com mais pessoas realizando meu exame.

$$
\text { Sim }
$$

Achei ruim porque aumenta o tempo da consulta.

$$
\text { Sim } 10
$$

Achei ruim porque perco minha privacidade.

Sim 26

Achei ruim porque sinto vergonha.

$$
\text { Sim } 55 \quad 73,3
$$

Achei ruim porque o aluno era do sexo masculino.

$$
\text { Sim } 22 \quad 29,3
$$

Achei ruim porque o exame realizado pelo aluno foi dolorido.

$$
\text { Sim }
$$

Meu sentimento é que posso contribuir com a formação médica.

$\operatorname{Sim}$ 156

De forma contraditória, a maioria, 187 (66,3\%), preferia ser submetida ao exame sem a presença do aluno, sendo esse sentimento predominante nas pacientes que consideravam como ruim o atendimento. A comparação encontra-se na Tabela 4.
TABELA 4

Comparação das respostas à questão "Você preferiria ser submetida ao exame sem a presença do aluno?

\begin{tabular}{lrrrrrr}
\hline $\begin{array}{l}\text { Questões do } \\
\text { questionário }\end{array}$ & $\mathrm{N}$ & $\%$ & $\mathrm{~N}$ & $\%$ & & \\
\hline \multicolumn{4}{l}{ Achei a presença do aluno boa. } \\
Não & 12 & 12,6 & 88 & 47,0 & 32,62 & 0,0 \\
Sim & 83 & 87,4 & 99 & 52,9 & & \\
Achei a presença do aluno ruim. & & & & \\
Não & 91 & 95,8 & 116 & 62,0 & 36,77 & 0,0 \\
Sim & 4 & 4,2 & 71 & 37,9 & & \\
\hline
\end{tabular}

(*) Resultado do teste de qui-quadrado de Pearson ou do teste exato de Fisher.

(**) Valor de P.

\section{DISCUSSÃO}

O presente trabalho foi realizado com mulheres atendidas nos ambulatórios de Ginecologia e Obstetrícia de uma cidade de Minas Gerais. O município conta com uma Faculdade de Medicina que está inserida nas esferas de atenção à saúde, principalmente na Atenção Primária. Os alunos desta instituição realizam atendimento, supervisionado pelos docentes, como parte obrigatória do aprendizado prático nos quatro pilares do ensino médico: Ginecologia e Obstetrícia, Cirurgia Geral, Clínica Médica e Pediatria.

Para compreender de que forma as mulheres percebem o atendimento ginecológico realizado pelo estudante, analisou-se o receio da paciente e a aceitação da presença do estudante, levando em consideração o sexo do mesmo e as justificativas para a aceitação ou não do acadêmico durante a consulta.

\section{Receio do atendimento realizado pelo estudante de Medicina (Tabela 1)}

Mais da metade das mulheres entrevistadas relataram ter receio quanto à presença do estudante durante o exame, sendo observados os fatores que contribuem para minimizar ou aumentar esse sentimento.

A informação prévia sobre a presença do estudante durante a consulta é um dos fatores que influenciam na aceitação. Neste estudo, a maioria relatou não ter sido previamente informada, e a ausência de informação aumentou a frequência de receio neste grupo. Embora sem significância estatística, os dados demonstram a mesma tendência da literatura. Um estudo americano demonstrou que poucos hospitais de ensino informavam a presença do aluno às pacientes e que menos de $50 \%$ das pacientes esperavam o envolvimento do estudante em sua consulta ${ }^{10}$. 
Observou-se também que a maioria das mulheres que relataram ter receio gostaria de ter recebido orientação prévia quanto à presença do aluno. Esta é vista com tanta importância que Berry estudou qual seria a melhor maneira de oferecer às pacientes esta informação e de lhes pedir permissão ${ }^{10}$. Percebeu que a maioria preferia ser informada por outro profissional que não o médico ou o estudante, para não haver pressão para aceitação ${ }^{10}$.

A necessidade de inserir o aluno na prática médica não pode extrapolar a autonomia das pacientes na escolha de quem irá prestar seu atendimento. Este estudo endossou a importância da conscientização dos personagens envolvidos de que a informação deve ser oferecida a todas as mulheres que procuram atendimento. Tal atitude é uma forma de demonstrar respeito e de aperfeiçoar a aceitação do estudante durante a consulta.

Observou-se também que, entre as mulheres que relataram ser boa a participação do aluno durante a consulta, a maioria não demonstrou receio. Semelhantemente à literatura, esse achado mostrou que pacientes com boas experiências prévias com estudantes têm maior disposição quanto ao envolvimento destes durante o atendimento ${ }^{1,2,3,9,13}$. Sob esta perspectiva, incentivar o primeiro contato da mulher com o acadêmico é recomendado, visto que experiências positivas minimizam o receio e reduzem a ansiedade que esse tipo de atendimento pode causar.

De forma contrária, a ausência ou poucas experiências prévias $^{3}$ e também experiências ruins ${ }^{2,9}$ influenciam negativamente a percepção das mulheres sobre o envolvimento do acadêmico ${ }^{2,3,9}$. No presente estudo, a maioria das mulheres que esperavam pelo primeiro atendimento no qual o estudante estaria presente se mostrou receosa em relação a esta consulta, relatando sentimentos negativos, como nervosismo, ansiedade, medo, desconforto, constrangimento e vergonha.

Verificou-se também que algumas mulheres relatavam sentimentos positivos, como tranquilidade e consciência da necessidade de participar na formação médica mesmo sem contato prévio com acadêmicos, e que esses sentimentos contribuíram para a menor frequência de receio. Esse aspecto pode refletir o perfil cultural dessa população, que há mais de uma década é assistida por meio de uma parceria entre a Secretaria Municipal de Saúde e a Faculdade de Medicina local, contando com a presença dos docentes e estudantes em todos os níveis de atenção à saúde. O caráter cultural é observado, também, em estudos internacionais como justificativa para melhor aceitação dos estudantes pela paciente ${ }^{1,9}$.

Neste estudo, foi observado que ter filhos contribuiu para menor receio. Mulheres que realizaram maior número de consultas, incluindo o acompanhamento pré-natal, se mostraram mais dispostas ao envolvimento do estudante no seu cuida$\mathrm{do}^{8,9}$. Ter filhos reflete maior número de experiências prévias ou, ainda, o amadurecimento da mulher, aumentando sua capacidade de compreensão.

Quase 90\% das mulheres que informaram ter receio em relação à presença do aluno durante a consulta diziam preferir ser submetidas ao exame apenas com o médico. Nesse contexto, surge a possibilidade de substituir pacientes por simulações ${ }^{12}$, No entanto, isto poderia tornar o custo do ensino médico muito alto e o sistema poderia colapsar ${ }^{12}$. Os manequins e simulações, entretanto, poderiam ser utilizados de forma complementar no aprendizado ${ }^{12}$ a fim de aprimorar a técnica do exame, diminuindo a insegurança do estudante e melhorando a aceitação das pacientes.

As pacientes precisam ser tranquilizadas em relação ao não comprometimento da qualidade do seu atendimento quando feito por estudantes ${ }^{12}$, visto que, em um cenário de ensino, a abordagem da mulher deve ser realizada de forma integral no que tange a anamnese, exame físico, propedêutica e orientação para a promoção da saúde. A atitude profissional, humana e técnica do acadêmico é uma ferramenta essencial para que a paciente perceba o caráter sério desse tipo de atendimento. Estas atitudes devem ser valorizadas durante o ensino, fazendo-se a ressalva de que o professor exerce papel fundamental nesse aspecto, por servir de exemplo ${ }^{9}$

Das mulheres que afirmaram não ter receio, mais da metade não se importa de ser atendida pelo estudante, com supervisão. Um estudo demonstra que o atendimento pelo acadêmico não diminui e pode até aperfeiçoar a qualidade da consulta, segundo a perspectiva da paciente ${ }^{1}$. Esse aspecto deve ser encarado pelos estudantes como fator estimulante ao aprendizado e também incentivador para o aperfeiçoamento contínuo do acolhimento, priorizando atender às necessidades das mulheres e corresponder as suas boas expectativas. Saber que as mulheres têm boa percepção do envolvimento acadêmico é gratificante.

Quando se analisa a necessidade de retorno para o atendimento realizado pelo estudante, o receio é desconsiderado pelas mulheres, uma vez que quase todas relataram que voltariam a consultar, resultado semelhante ao da literatura ${ }^{3}$. No entanto, percebeu-se que, considerando o perfil socioeconômico desta população, muitas delas voltariam a consultar por falta de opção e não necessariamente porque gostaram do atendimento realizado. No entanto, esse dado não foi objeto de pesquisa.

\section{Aceitação da paciente em relação ao sexo do aluno (Tabela 2)}

O sexo do aluno que presta assistência nos ambulatórios de Ginecologia e Obstetrícia, em especial o masculino, pode re- 
presentar uma barreira adicional na relação entre os estudantes de Medicina e as mulheres atendidas².

No presente trabalho, mais da metade das entrevistadas relataram que o sexo do aluno não influencia na sua aceitação do estudante. Esse resultado acompanha o mesmo achado de Ching ${ }^{7}$.

Dentre aquelas que afirmaram que o sexo do aluno gera receio quanto à sua participação durante a consulta, a grande maioria preferia ser atendida pelas estudantes. Outros autores evidenciaram resultados semelhantes ${ }^{2,4,9}$. O sexo feminino foi considerado fator determinante para que algumas mulheres permitissem a participação do estudante durante a consulta ${ }^{3,4}$, e o masculino como justificativa para a recusa do atendimento em um terço dos casos ${ }^{2}$. Rizk relatou que a minoria das mulheres se dizia preparada para ter estudantes do sexo masculino envolvidos em seu atendimento e sugere que alunos desse gênero poderiam utilizar manequins e simulações como alternativas à recusa das mulheres ${ }^{9}$.

Ching observou que os homens percebiam maior recusa por parte das mulheres ao seu atendimento quando comparados às estudantes ${ }^{7}$. Para evitar que o estudante do sexo masculino tenha seu aprendizado prático prejudicado, uma boa opção é formar grupos mistos, minimizando-se, assim, o incômodo que a diferença de sexo entre estudante e paciente pode causar.

Independentemente do sexo do aluno que havia prestado atendimento, as pacientes não relataram diferença quanto ao interesse demonstrado pelo aluno e às orientações dadas a elas. $\mathrm{O}$ aluno ser do sexo feminino foi determinante no sentimento de conforto relatado pelas mulheres, e o mesmo aspecto foi encontrado por Rizk, especialmente quando as queixas eram de caráter sexual ou quando exames pélvicos eram realizados ${ }^{9}$.

Entre aquelas que acharam que o exame realizado foi mais completo ou que sentiram maior segurança com mais pessoas realizando seu exame, a maior porcentagem foi encontrada no grupo que havia sido atendido exclusivamente por acadêmicos do sexo masculino. Resultado semelhante foi encontrado dentre as que consideraram que foram mais bem atendidas quando apenas o estudante do gênero masculino estava participando, entretanto sem diferença estatisticamente significativa. Segundo Roter, os ginecologistas apresentam níveis mais altos, mas não significativos, de conversação emocional com suas pacientes do que as médicas da mesma especialidade, sendo um ponto crucial para aceitação e bom relacionamento ${ }^{14}$.

Esses achados são fundamentais para demonstrar que a especialidade de Ginecologia e Obstetrícia não pode ser de- sencorajada para o sexo masculino ${ }^{8}$ e que, apesar de o número de homens especialistas nessa área estar diminuindo, a maioria das mulheres não mostra preferência pelo sexo do profissional que a acompanha ${ }^{15}$. A adoção de uma postura profissional madura e a realização de um acolhimento adequado são fatores que reduzem a distância entre o estudante do gênero masculino e a paciente.

O sexo do aluno não foi significativo para determinar nenhuma das justificativas das mulheres que consideraram ruim o atendimento pelo estudante. Entretanto, dentre as que relataram vergonha, a frequência foi maior nas mulheres que haviam sido atendidas por estudantes exclusivamente do sexo feminino. Percebeu-se, portanto, que, mesmo que o aluno seja do sexo feminino, sua presença pode implicar sentimentos negativos, talvez por outros motivos, como, por exemplo, número excessivo de estudantes que participam da consulta ${ }^{2}$, postura perante a paciente, perfil tímido da mulher e também o caráter mais íntimo da consulta ginecológica.

\section{Justificativas para aceitação ou não do estudante pela paciente durante a consulta (Tabelas 3 e 4)}

Neste estudo, a maioria das pacientes preferia ser submetida ao exame ginecológico exclusivamente com o médico. Essa relação foi mais importante nas mulheres que consideraram ruim o atendimento pelo aluno. Esses dados são diferentes daqueles da literatura, que mostra que a minoria das pacientes preferia o atendimento exclusivo com o médico ${ }^{9,11}$. Essa diferença pode ser explicada pelo fato de que, nos vários estudos realizados em populações estrangeiras, as próprias mulheres buscavam esse tipo de atendimento por entenderem a importância da vivência prática para o aluno, ao passo que no presente estudo a maioria das pacientes pesquisadas recorria a este atendimento por ser o único a que tinha acesso.

Este estudo mostrou que as principais justificativas para a rejeição ao aluno durante a consulta foram o relato de vergonha e perda de privacidade. Também foram relatadas outras justificativas, como aumento do tempo da consulta, desconforto pelo aluno ser do sexo masculino e o exame ter sido dolorido. A literatura, além de apresentar razões semelhantes, como aumento do tempo da consulta e desejo de privacida$\mathrm{de}^{3,9,11}$, mostra outras justificativas: repetição de atividades, inexperiência dos estudantes, desejo de falar em particular com o médico ${ }^{3}$ e incerteza quanto ao grau de envolvimento do estudante durante o exame ${ }^{9}$. Apesar dos aspectos negativos, é preciso ressaltar que o estudante só aprende com a prática, e a inexperiência é natural durante o aprendizado.

Das mulheres que relataram achar bom o atendimento pelo aluno durante a consulta, a maioria não se importava de 
ser examinada por ele. A aceitação das pacientes é justificada principalmente pelo maior interesse demonstrado pelos alunos, pela maior orientação que elas recebem e pelo exame ser mais completo. A literatura apresenta resultados semelhantes $^{1,3,9}$. Durante o atendimento realizado pelo estudante, cada paciente representa uma nova oportunidade para o aprendizado, o que faz com que o aluno dispense mais atenção à mulher e realize um exame completo, visando extrair o máximo possível de informações.

Neste estudo, a possibilidade de contribuir para a formação médica foi a principal percepção que a mulher teve em relação ao atendimento pelo estudante. Esse dado foi concordante com a literatura, que relata que esta é a motivação primária para a aceitação do atendimento realizado pelo estudante ${ }^{3,9,11}$. Esse é um aspecto positivo, uma vez que mostra que, independentemente dos incômodos causados pela presença dos estudantes durante a consulta ginecológica, a maioria das mulheres tem consciência da importância de sua participação no aprendizado médico.

Uma limitação apresentada pelo trabalho foi a avaliação restrita das mulheres que frequentaram os ambulatórios de Ginecologia e Obstetrícia ofertados pelo Sistema Único de Saúde (SUS) e são mais habituadas à presença do estudante ou têm dificuldade em conseguir consulta exclusiva com o médico, gerando o questionamento da aceitação do atendimento por falta de opção ou por preferência. Aquelas que não frequentam o ambulatório, independentemente da motivação, não foram abordadas, e os valores de receio encontrados podem estar subestimados.

\section{CONCLUSÃO}

O presente estudo identificou que, na população avaliada, a maior parte das mulheres relatou como boa a participação do aluno durante seu atendimento, e o interesse demonstrado pelos mesmos foi a principal justificativa para essa percepção. Não houve preferência com relação ao gênero do estudante que havia prestado assistência. Entretanto, observou-se que as mulheres ainda apresentam receio de serem examinadas por estudante e que, apesar do sentimento positivo em relação ao estudante, a maioria prefere o atendimento realizado exclusivamente pelo médico.

Como contribuição para melhorar a relação estudante-mulher durante o atendimento ginecológico, ficam as seguintes sugestões: as instituições de ensino médico devem evitar grupos com número excessivo de alunos, preconizando o menor número possível por sala; mesclar alunos de gêneros diferentes na tentativa de evitar o constrangimento causado pela diferença de sexo. As unidades básicas de saúde devem se certificar de que as mulheres estão sendo informadas sobre a presença do aluno durante a consulta. $\mathrm{O}$ acadêmico deve ter postura acolhedora, séria, comprometida e profissional durante o atendimento, cabendo ao professor dar o exemplo e exigir dos alunos o mesmo comportamento. Finalmente, deve haver maior participação dos membros das equipes de saúde para tornar este processo mais eficaz.

\section{REFERÊNCIAS}

1. Cooke F, Galasko G, Ramrakha V, Duncan R, Rose A, Watkins J. Medical students in general practice: how do patients feel? Brit J Gen Practice. 1996;46:361-62.

2. Fortier AM, Hahn PH, Trueman J, Reid RL. The acceptance of medical students by women with gynecology appointments. J Obstet Gynaecol Cam. 2006;28(6):526-30.

3. Hartz MB, Beal JR. Patient's attitudes and comfort levels regarding medical students` involvement in obstetrics-gynecology outpatient clinics. Acad Med. 2000;75(10):101014.

4. O'Flynn N, Rymer J. Women's attitudes to the sex of medical students in a gynaecology clinic: cross sectional survey. BMJ. 2002;325:683-84.

5. Brasil. Ministério da Educação. Conselho Nacional de Educação. Câmara de Educação Superior. Resolução CNE/ CES n. ${ }^{\circ}$ 4, de 7 de novembro de 2001. Institui diretrizes curriculares nacionais do curso de graduação em medicina. Diário Oficial da União. Brasília, 9 nov. 2001; Seção 1, p.38.

6. Almeida FCM, MacielI APP, Bastos AR, Barros FC, Ibiapina JR, Souza SMF, Araújo DP. Avaliação da inserção do estudante na unidade básica de saúde: visão do usuário. Rev Bras Educ Med. 2012;36(1):33-9.

7. Ching SL, Gates EA, Robertson PA. Factors influencing obstetric and gynecologic patients decisions toward medical student involvement in the outpatient setting. Am J Obstet Gynecol. 2000;182(6):1429-32.

8. Racz JM, Srikanthan A, Hahn PM, Reid RL. Gender preference for a female physician diminishes as women have increased experience with intimate examinations. J Obstet Gynaecol Cam. 2008;30(10):910-17.

9. Rizk DEE, Al-Shebah A, El-Zubeir MA, Thomas LB, Hassan MY, Ezimokhai M. Women's perceptions of and experiences with medical student involvement in outpatient obstetric and gynecologic care in the United Arab Emirates. Am J Obstet Gynecol. 2002;187(4):1091-100.

10. Berry RE, O`Dell K, Meyer BA, Purwono U. Obtaining patient permission for student participation in obstetric-gynecologic outpatient visits: a randomized controlled trial. Am J Obstet Gynecol. 2003;189(3):634-8. 
11. Simons RJ, Imboden E, Martel JK. Patients attitudes towards medical students participation in a general internal medicine clinic. J Gen Intern Med. 1995;10:251-4.

12. Lowe M, Kerridge I, McPhee J, Hart Clare. Do patients have an obligation to participate in student teaching? Med Educ. 2008;42:237-41.

13. Magrane D, Gannon J, Miller CT. Obstetric patients who select and those who refuse medical students' participation in their care. Acad Med. 1994;69:1004-6.

14. Roter DL, Hall JA, Aoki Y. Physician gender effects in medical communication. JAMA 2002;288(6):756-64.

15. Johnson AM, Schnatz PF, Kelsey AM, Ohannessian CM. Do women prefer care from female or male obstetrician-gynecologists? A study of patient gender preference. Med Educ. 2005;105(8):369-72.

\section{CONTRIBUIÇÃO DOS AUTORES}

Suzana Maria Pires do Rio: orientação, coordenação, análise e interpretação de dados, revisão crítica do conteúdo, aprovação final da versão do estudo. Ivana M. Trivellato: aquisição de dados, análise e interpretação de dados, elaboração da versão inicial do artigo e revisão crítica do conteúdo, aprovação da versão final do estudo encaminhado para publicação. Natália M. Caldeira: aquisição, análise e interpretação de dados, elaboração da versão inicial do artigo elaboração da versão inicial do artigo e revisão crítica do conteúdo, aprovação da versão final do estudo encaminhado para publicação. Sílvia F. Araujo: aquisição de dados, análise e interpretação de dados, elaboração da versão inicial do artigo e revisão crítica do conteúdo, aprovação da versão final do estudo. Dilermando Fazito: contribuição para concepção do desenho do estudo, cálculo amostral e banco de dados, análise e interpretação de dados, elaboração da versão inicial do artigo e revisão crítica do conteúdo e aprovação da versão final do estudo.

\section{CONFLITO DE INTERESSES}

Declarou não haver.

\section{ENDEREÇO PARA CORRESPONDÊNCIA}

Silvia Ferreira Araujo

Av. do Contorno, 8671 apt. 101

Gutierrez - Belo Horizonte

CEP. 30110-059 MG

E-mail : silvis_fa@hotmail.com 